\title{
MicroRNAs in prostate cancer
}

\author{
Won Tae Kim, Wun-Jae Kim \\ Department of Urology, Chungbuk National University College of Medicine, Cheongju, Korea
}

\begin{abstract}
MicroRNAs (miRNAs) are made up of -22 endogenous nucleotides and are small, noncoding RNAs that are important regulators of gene expression at the posttranscriptional level by degrading or repressing target miRNAs. miRNA expression profiles can be used for the detection of diagnostic and prognostic markers for various cancers. Also, alterations of miRNAs in cancer tissues have been associated with clinicopathological parameters. Along with circulating miRNAs, tissue miRNAs have shown promise as markers that can predict cancer recurrence and/or the potential for survival of cancer patients. Additionally, some miRNAs have therapeutic potential. In this review, we discuss and assess the usefulness of tissue-derived and circulating miRNAs for the diagnosis and prognosis of prostate cancer.
\end{abstract}

Keywords: Prostatic neoplasms, MicroRNAs, Biological markers

\section{INTRODUCTION}

Prostate cancer is the most common form of cancer for males in Western countries, and the incidence is increasing in many of those countries [1]. Early diagnosis of cancer and timely detection of disease progression following either radical prostatectomy or radiation therapy are crucial for the effective treatment of prostate cancer and for a beneficial clinical outcome. To increase the detection rate of early-stage prostate cancer, there has been much research to develop effective biomarkers. Currently, measurement of serum prostate-specific antigen (PSA) is the most common tool used to detect prostate cancer. PSA levels correlate with prostate cancer risk, aggressiveness, and outcome [2]. Although PSA is considered to be the most useful biomarker for prostate cancer [3,4], it has limitations such as a low detection rate for prostate cancer in the so-called gray zone (PSA, 4-10 ng/mL) and no advantage of PSA screening for a control group [5]. Moreover, the benefit of mass screening by PSA testing and the establishment of systemized surveillance protocols according to PSA levels are still under debate. Also, although PSA screening has reduced the mortality rate of prostate cancer, it is often associated with over-diagnosis [6]. Thus, there has been rapidly growing interest in alternative biomarkers for prostate cancer, such as microRNAs (miRNAs).

miRNAs are made up of -22 endogenous nucleotides and are small, noncoding RNAs that are important regulators of gene expression at the post-transcriptional level because they degrade, or repress, target mRNAs [7]. miRNAs could play important roles in various processes such as cell development, differentiation, proliferation, cell-cycle control, apoptosis, and metabolism [7]. The expression of miRNAs can be influenced by chromosomal rearrangements (deletions, amplifications, and mutations), promoter methylation, and regulation of transcription. Aberrations can result in miRNA up- and down-regulation. Additionally, a single miRNA can target hundreds of mRNAs. Hence, miRNA alterations are known to disrupt the expression of several mRNAs and proteins, and can be involved in the occurrence of various human cancers $[8-10]$.

Corresponding author: Wun-Jae Kim

Department of Urology, Chungbuk National University College of Medicine, 52 Naesudong-ro, Heungdeok-gu, Cheongju 361-804, Korea

E-mail: wjkim@chungbuk.ac.kr / Tel: +82-43-269-6371 / Fax: +82-43-269-6144

Submitted: 12 December 2012 / Accepted after revision: 13 December 2012

Copyright @ $\odot 2013$ Asian Pacific Prostate Society (APPS) 
There have been many reports about disease-specific miRNA expression profiles in various human cancers [1116]. The miRNA expression profiling of human cancers is increasing in importance due to its usefulness in diagnosis, staging, progression, prognosis, and response to treatment. Consequently, miRNAs are being regarded as new oncogenes or tumor suppressor genes and new biomarkers for the diagnosis and prediction of treatment response and prognosis. Recently, several studies have demonstrated that miRNAs are sufficiently stable after being subjected to severe conditions, such as boiling, very low or high $\mathrm{pH}$, extended storage, and several freeze-thaw cycles, and they are well protected from Ribonuclease in blood serum and plasma, as well as from exposure to other body fluids [17-19]. Therefore, stability makes miRNA levels well suited for testing in patient samples. In this review, we discuss and assess the usefulness of tissue-derived and circulating miRNAs for the diagnosis and prognosis of prostate cancer.

\section{miRNAs AS BIOMARKERS IN SOLID TUMORS}

miRNA expression profiles in various cancer tissues can be diagnostic and prognostic markers of each cancer. Several studies have demonstrated the up- and down-regulation of miRNAs associated with cancer pathogenesis $[14,16]$. He et al. [14] found that three miRNAs (miR-221, miR-222, and miR-146) were transcriptionally up-regulated in papillary thyroid carcinoma compared to normal thyroid tissue. They suggested that the up-regulation of these miRNAs is involved in thyroid carcinogenesis. Also, Volinia et al. [16] demonstrated 21 miRNAs that were up-regulated in six solid cancer tissues. They found predicted targets, which included the tumor suppressing genes $R B 1$ and TGFBR2. They suggested that miRNAs are extensively involved in the tumorigenesis of solid tumors and that they support this function as either dominant or recessive cancer genes.

Alterations of miRNA in cancer tissues have been associated with the clinicopathological parameters of each cancer. Yanaihara et al. [15] showed that miRNA expression profiles of lung cancer were correlated with the survival of lung cancer patients. Both high miR-155 and low miR-let-7a-2 expression are associated with poor survival. Murakami et al. [13] showed that three miRNAs (miR-224, miR-18, and precursor miR-18) demonstrated a higher expression in the hepatocellular carcinoma (HCC) samples, and that five miRNAs (miR199a, miR-199a*, miR-200a, miR-125a, and miR-195) demonstrated a lower expression in the HCC samples in miRNA expression profiles between HCC and nontumorous tissue. They also reported expression levels of some miRNAs that were inversely correlated with the degree of HCC differentiation. Iorio et al. [12] demonstrated miRNA expressions of miR-125b, miR-145, miR-21, and miR-155 that were deregulated significantly in miRNA expression profiles of breast cancer tissues, and they identified miRNAs with expressions that were correlated with specific breast cancer biopathologic features such as estrogen and progesterone receptor expression, tumor stage, vascular invasion, or proliferation index. In this regard, miRNA expression profiles of cancer tissues could be postulated as useful markers for the diagnosis and prognosis of various cancer patients.

Recently, tumor-derived circulating exosomes, including miRNAs in ovarian cancer, have shown similarities with cellular miRNAs [20]. Moreover, in another study, circulating exosomal miRNAs have been correlated with tumor miRNA levels in lung cancer [21]. These results suggest that circulating exosomal miRNAs could potentially be used as diagnostic markers for screening in asymptomatic populations. More recently, circulating miRNAs were analyzed for promising markers to detect early cancers such as colon cancer and pancreatic cancer $[22,23]$. When comparing the miRNAs in plasma between cancer patients and healthy controls, researchers found novel noninvasive biomarkers (miR-29a and miR-92a in colon cancer and miR-210 in pancreatic cancer) that were useful for early detection. Resnick et al. [24] demonstrated that circulating miRNAs (miR-21, miR-92, and miR93) as diagnostic markers in ovarian cancer might be more feasible than established serum-based screening markers such as CA-125. These results indicate that circulating miRNAs might be noninvasive diagnostic markers compared with established clinical diagnostic tests that are invasive.

It is noteworthy that circulating miRNAs could be a useful marker for follow-up after treatment. Yamamoto et al. [25] found that the expression of miR-500, an oncofetal miRNA, changed during liver development in liver cancer. They also showed that miR-500 was abundantly expressed in $45 \%$ of human HCC tissue. Notably, in the sera of HCC patients, the amount of miR-500 was increased. Furthermore, elevated miR-500 in HCC patients returned to normal serum levels after surgery. These results reveal that the miR-500 in the serum of the HCC patients might be an oncofetal miRNA that reflects pathological conditions. In addition, Wong et al. [26] demonstrated that miR-184 in squamous cell carcinoma also acted similar to miR-500 in HCC. Feng et al. [27] also showed that circulating RNA levels in renal cell carcinoma patients were significantly higher than normal controls and were re- 
duced after nephrectomy. Several reports have suggested that the identified circulating miRNAs might be valuable biomarkers for cancer detection [24,28]. However, miRNAs in tissues show promise as markers that can predict cancer recurrence and the survival of cancer patients $[29,30]$.

\section{miRNAS IN PROSTATE CANCER}

Molecular alterations such as chromosomal instability, epigenetic silencing, alteration of tumor-suppressor genes, and some protein expressions are associated with the tumorigenesis of prostate cancer [31,32]. Furthermore, aberrant electroretinogram (ERG) expression, loss of phosphatase and tensin homolog (PTEN), and inactivation of CD9 promotes the progression of prostate cancer [33,34]. In addition, androgen receptor signaling is very important in the progression to castration-resistant prostate cancer [35]. Interestingly, these genetic alterations in cancer play important roles in the specific activity of miRNA. In prostate cancer, $30 \%$ of epigenetic silencing regions contained the loci of miRNAs [36]. DNA methylation of a promoter region and $p 53$ mutation leads to a down-regulation of miRNA [37]. Sun et al. [38] reported similar results. They analyzed the expression patterns of miRNA target genes in public microarray datasets. They inferred a general overexpression of miRNAs in prostate cancer based on the reduced abundance of putative miRNA targets in human prostate cancer. Furthermore, Xu et al. [39] found that functional polymorphism in the pre-miR-146a of prostate cancer tissue affects the amount of miR-146a, and is associated with prostate cancer risk.

The expression of specific miRNAs could be a classifier that could be used to differentiate between normal and cancer tissues. However, differences in the expression patterns of miRNAs that have been reported in different studies, which creates controversy in their use as classifiers. In fact, Volinia et al. [16] demonstrated that miR-21, miR-17-5p, miR-191, miR29b-2, miR-199a-1, miR-146, miR-181b-1, miR-20a, miR-32, miR-92-2, miR-214, miR-30c, miR-25, and miR-106a all were up-regulated in prostate cancer. Those researchers verified a general up-regulation of miRNAs in cancer tissue. However, 6 miRNAs were down-regulated in their study. Conversely, Porkka et al. [40] observed a down-regulation for 37 miRNAs and an up-regulation for 14 miRNAs in prostate cancer. In the Porkka study, they suggested that genetic aberrations might target miRNAs and found that miRNA expression in lymph nodes could be classified according to their androgen dependence. In addition, Ozen et al. [41] found widespread downregulation of miRNAs in prostate cancer tissues. In the studies of Porkka et al. [40] and Ozen et al. [41], the let-7 family, miR16, miR-23, miR-99, miR-125, miR-29, and miR-30 all were down-regulated. Ambs et al. [42] found that prostate cancer cells expressed higher miR-32 levels than normal prostate cells. They demonstrated that miR-32, miR-26a, miR-196a, miR-181a, miR-25, miR-93, miR-92, and let-7i all were upregulated and also identified some miRNAs associated with extraprostatic disease extension, androgen regulation, and transcription of protein-coding target genes. These results partially agreed with the data found by Volinia et al. [16].

miRNAs in prostate cancer tissues might be promising biomarkers that can be used to detect cancer compared to normal controls. Szczyrba et al. [43] demonstrated that 33 miRNAs in prostate cancer tissues either were up-regulated or down-regulated more than 1.5-fold compared to that found in noncancer tissues. They suggested that miR-143 and miR145 can be involved in the development of prostate cancer. Furthermore, Mattie et al. [44] showed that miRNA in biopsy specimens in prostate cancer patients could be used to separate prostate cancer from normal controls by using their highthroughput miRNA expression profiling.

miRNAs in cancer tissues might be a useful marker for the prognosis of prostate cancer. Tong et al. [45] performed paired analysis of 40 prostatectomy specimens (malignant vs. noninvolved areas of each specimen) including 20 with biochemical recurrence within 2 years after prostatectomy and 20 without a recurrence for more than 10 years. They demonstrated that 5 miRNAs (miR-23b, miR-100, miR-145, miR221, and miR-222) were down-regulated in cancer tissues, which agreed with the results found by Porkka et al. [40]. Also, the expressions of miR-135b and miR-194 were increased in cancer tissues with early biochemical recurrence. Schaefer et al. [46] also investigated the associations of miRNA expression and clinicopathological data in matches between 76 prostate cancer tissue samples and samples of adjacent normal tissues. They identified 10 miRNAs such as miR-16, miR31, miR-125b, miR-145, miR-149, miR-181b, miR-184, miR205, miR-221, and miR-222 that were down-regulated and 5 miRNAs such miR-96, miR-182, miR-182*, miR-183, and miR375 that were up-regulated. Some miRNA expressions correlated with the Gleason score and the tumor stage. miR-96 expression was associated with a recurrence after surgery. In addition, Spahn et al. [47] also analyzed the global expression of miRNAs in benign prostatic hyperplasia (BPH) and metastatic prostate cancer. They found that some miRNAs are oncomirs and that down-regulation of miR-221 was associated with the Gleason score, progression, and recurrence. Furthermore, miRNA signatures in prostate cancer tissues could 
be a prognostic marker that could be used to independently forecast postoperative outcome in later disease stages [48].

Similarly, several studies of mRNA expression signatures in cancer tissues have been associated with prognostic parameters. Cuzick et al. [49] assessed the prognostic value of the mRNA expressions of 31 genes involved in cell-cycle progression in prostatectomy samples. They demonstrated that cell-cycle progression scores and PSA concentration were the most important predictors of recurrence, survival, and prognosis. In addition, Penney et al. [50] found that 157 mRNA expression signatures in prostate cancer tissues could be used to predict both the Gleason score and the relative risk of lethality, which was helpful in the avoidance of overtreatment.

\section{miRNAs IN THE BODY FLUIDS OF PROS- TATE CANCER PATIENTS}

In the past, researchers suspected that miRNAs were relevant to the regulation of cancer-associated genes in tissues. In 2008, Lawrie et al. [51] first identified the fact that cell-free miRNAs in body fluids such as serum might have potential as noninvasive diagnostic markers. They found that the expression levels of miR-155, miR-210, and miR-21 in serum from diffuse large B-cell lymphoma patients were significantly higher than that found in normal controls. Since then, there has been growing interest in circulating miRNAs. Chen et al. [18] showed that cell-free miRNAs are present, stable, reproducible, and consistent in the serum and plasma of humans. In addition, they found that each disease such as lung cancer, colorectal cancer, and diabetes has a specific expression pattern of serum miRNAs. Thus, detection of miRNAs in body fluids could serve as potential noninvasive diagnostic biomarkers of various cancers.

In 2008, Mitchell et al. [19] reported that tumor-derived miRNAs can enter the circulatory system and can be measured in serum and plasma as important blood-based biomarkers of human cancer. They also showed that circulating miR-141 was significantly elevated in the sera of prostate cancer patients when compared with healthy controls. Their report was the first description of circulating miRNA in patients with metastatic prostate cancer. Recently, the up-regulation of miR-141 in the plasma of metastatic prostate cancer patients was confirmed [52]. Brase et al. [53] also found that circulating miRNAs (miR-375 and miR-141) were highly detected in patients with advanced disease, and their levels also correlated with a high Gleason score or with a positive status for the lymph-node. In addition, miRNAs in tumors were sig- nificantly up-regulated compared to normal tissue. These results suggested that some miRNAs including miR-141 might be the most promising markers correlated with prostate tumor progression. The function of miR-141 in prostate cancer cells is poorly understood. Waltering et al. [54] identified that miR-141 was up-regulated after castration, and its activation induced the increased growth of LNCaP cells. It is possible that miR-141 regulates androgen, which plays a critical role in the growth of both androgen-dependent and castration-resistant prostate cancer. Therefore, miR-141 may be functionally involved in the disease progression of prostate cancer.

Circulating miRNAs might be associated with specific clinicopathological parameters, risk, aggressiveness, staging, and disease outcomes. For instance, Mahn et al. [55] analyzed circulating miRNA in the serum of patients with localized prostate cancer, $\mathrm{BPH}$ and healthy controls. They found that miR-195 correlated with clinicopathological parameters such as surgical margin positivity and the Gleason score, and that miR-let7i also correlated with some specific parameters. In addition, Shen et al. [56] investigated the plasma miRNAs of 82 prostate cancer patients to analyze the association between miRNAs and the development and progression of prostate cancer. They found that some miRNAs could distinguish lowvs. intermediate- vs. high-risk patients of prostate cancer and could predict the aggressiveness of prostate cancer. Furthermore, Bryant et al. [57] investigated circulating miRNAs for their usefulness as biomarkers for the diagnosis, staging, and prediction of outcomes. They analyzed the serum and urine of prostate cancer patients and demonstrated that some miRNAs were associated with metastasis in prostate cancer. They showed that circulating miRNAs could be diagnostic markers in urine samples. Interestingly, Lodes et al. [58] found that in as little as $1 \mathrm{~mL}$ of serum, sufficient miRNAs were present to detect prostate cancer and to discriminate between normal and prostate cancer patients. These results show that miRNAs can be measured and detected as biomarkers in small amounts of body fluids. Consequently, circulating miRNA expression, according to alterations, may be useful biomarkers for clinical diagnosis and prognosis.

\section{PROBLEMS WITH USING miRNAS}

miRNAs are one of the most important mediators for tumorigenesis, disease progression, and metastasis. Because miRNAs are mediated by gene signaling such as deletions, amplifications, mutations, and epigenetic alterations of DNA, they can affect the synthesis of proteins necessary to develop cancer, disease progression, and metastasis to other organs. 
Hence, miRNAs can be useful clinical diagnostic and prognostic biomarkers. Nevertheless, there are several limitations to be overcome before they can be used to replace conventional biomarkers such as PSA, alpha-fetoprotein (AFP), and carcinoembryonic antigen (CEA). Of course, established biomarkers also have limitations in distinguishing cancer and benign disease. Certainly, cancer biomarkers including CEA (colon cancer), AFP (HCC), and PSA are also highly detected in benign conditions. For example, PSA levels are increased in patients with prostatitis and $\mathrm{BPH}$.

First, there is no established endogenous ('housekeeping') miRNA control to normalize for miRNA levels in body fluids. U6 small nuclear RNA is usually used to normalize miRNA levels measured in tissues and body fluids. However, this miRNA control was found to be somewhat unstable compared to some miRNAs and was degraded in serum $[18,59]$. Some studies have used miR-16 and synthetic versions of Caenorhabditis elegans miRNAs [19,24]. However, measurements of miR-16 in serum might be inconsistent. Thus, to identify more stable controls for normalization, more studies and empirical validation are necessary. Second, the mechanism responsible for the release of miRNAs into body fluids, such as blood and urine, and its functional role and consequence is poorly understood. Only a few studies have examined the characteristics and functions of miRNAs in body fluids. For example, exosome that contains miRNAs can be delivered to other cells and miRNA-protein complexes could be the mechanism responsible for the stability of miRNAs in body fluids $[60,61]$. In particular, in cancer patients, tumor-derived microvesicles containing miRNAs stimulate angiogenesis, tumor progression, and metastasis [62]. Therefore, further extensive studies are necessary to identify the roles and consequences of miRNAs. Third, there have been inconsistencies in the analysis results of some miRNAs in various cancers. We hope that advances in science and highthroughput technology can solve these problems.

\section{FUTURE DIRECTIONS}

Interest is increasing in the use of miRNAs as diagnostic and prognostic biomarkers for cancer treatment. Many studies have identified and confirmed the use of miRNAs as biomarkers. Furthermore, some miRNAs have therapeutic potential in cancer patients [63]. Thus, some researchers are exploring the potential for miRNAs as therapeutic molecules.

There are many unexplored fields concerning miRNAs and prostate cancer and many topics for research in this field. Studies of miRNAs in urine have recently increased. Current approaches to the detection of cancer and to the prediction of its outcome for patients are invasive and unable to detect the early stages. Certainly, many studies are necessary to establish miRNAs as useful diagnostic biomarkers in blood and urine, and as prognostic biomarkers in cancer tissues. In the near future, miRNAs in tissues, blood, and urine will undoubtedly be established as new biomarkers for the diagnosis and prediction of treatment outcomes in prostate cancer.

\section{CONFLICT OF INTEREST}

No potential conflict of interest relevant to this article was reported.

\section{ACKNOWLEDGMENTS}

This research was supported by Basic Science Research Program through the National Research Foundation of Korea (NRF) funded by the Ministry of Education, Science and Technology (2012-0000476) and by a grant from the NextGeneration BioGreen 21 Program (No.PJ0081952011), Rural Development Administration, Republic of Korea.

\section{REFERENCES}

1. Gronberg H. Prostate cancer epidemiology. Lancet 2003;361: 859-64.

2. Loeb S, Catalona WJ. Prostate-specific antigen in clinical practice. Cancer Lett 2007;249:30-9.

3. Wang MC, Valenzuela LA, Murphy GP, Chu TM. Purification of a human prostate specific antigen. Invest Urol 1979;17:15963.

4. Oesterling JE. Prostate specific antigen: a critical assessment of the most useful tumor marker for adenocarcinoma of the prostate. J Urol 1991;145:907-23.

5. Andriole GL, Crawford ED, Grubb RL 3rd, Buys SS, Chia D, Church TR, et al. Mortality results from a randomized prostate-cancer screening trial. N Engl J Med 2009;360:1310-9.

6. Schroder FH, Hugosson J, Roobol MJ, Tammela TL, Ciatto S, Nelen V, et al. Screening and prostate-cancer mortality in a randomized European study. N Engl J Med 2009;360:1320-8.

7. Bartel DP. MicroRNAs: genomics, biogenesis, mechanism, and function. Cell 2004;116:281-97.

8. Calin GA, Croce CM. MicroRNA signatures in human cancers. Nat Rev Cancer 2006;6:857-66.

9. Chen CZ. MicroRNAs as oncogenes and tumor suppressors. N Engl J Med 2005;353:1768-71.

10. Caldas C, Brenton JD. Sizing up miRNAs as cancer genes. Nat 
Med 2005;11:712-4.

11. Calin GA, Liu CG, Sevignani C, Ferracin M, Felli N, Dumitru $\mathrm{CD}$, et al. MicroRNA profiling reveals distinct signatures in $\mathrm{B}$ cell chronic lymphocytic leukemias. Proc Natl Acad Sci U S A 2004;101:11755-60.

12. Iorio MV, Ferracin M, Liu CG, Veronese A, Spizzo R, Sabbioni $\mathrm{S}$, et al. MicroRNA gene expression deregulation in human breast cancer. Cancer Res 2005;65:7065-70.

13. Murakami Y, Yasuda T, Saigo K, Urashima T, Toyoda H, Okanoue $\mathrm{T}$, et al. Comprehensive analysis of microRNA expression patterns in hepatocellular carcinoma and non-tumorous tissues. Oncogene 2006;25:2537-45.

14. He H, Jazdzewski K, Li W, Liyanarachchi S, Nagy R, Volinia S, et al. The role of microRNA genes in papillary thyroid carcinoma. Proc Natl Acad Sci U S A 2005;102:19075-80.

15. Yanaihara N, Caplen N, Bowman E, Seike M, Kumamoto K, Yi M, et al. Unique microRNA molecular profiles in lung cancer diagnosis and prognosis. Cancer Cell 2006;9:189-98.

16. Volinia S, Calin GA, Liu CG, Ambs S, Cimmino A, Petrocca $\mathrm{F}$, et al. A microRNA expression signature of human solid tumors defines cancer gene targets. Proc Natl Acad Sci U S A 2006;103:2257-61.

17. Yun SJ, Jeong P, Kim WT, Kim TH, Lee YS, Song PH, et al. Cell-free microRNAs in urine as diagnostic and prognostic biomarkers of bladder cancer. Int J Oncol 2012;41:1871-8.

18. Chen X, Ba Y, Ma L, Cai X, Yin Y, Wang K, et al. Characterization of microRNAs in serum: a novel class of biomarkers for diagnosis of cancer and other diseases. Cell Res 2008;18:9971006.

19. Mitchell PS, Parkin RK, Kroh EM, Fritz BR, Wyman SK, Pogosova-Agadjanyan EL, et al. Circulating microRNAs as stable blood-based markers for cancer detection. Proc Natl Acad Sci U S A 2008;105:10513-8.

20. Taylor DD, Gercel-Taylor C. MicroRNA signatures of tumorderived exosomes as diagnostic biomarkers of ovarian cancer. Gynecol Oncol 2008;110:13-21.

21. Rabinowits G, Gercel-Taylor C, Day JM, Taylor DD, Kloecker GH. Exosomal microRNA: a diagnostic marker for lung cancer. Clin Lung Cancer 2009;10:42-6.

22. Huang Z, Huang D, Ni S, Peng Z, Sheng W, Du X. Plasma microRNAs are promising novel biomarkers for early detection of colorectal cancer. Int J Cancer 2010;127:118-26.

23. Ho AS, Huang X, Cao H, Christman-Skieller C, Bennewith K, Le QT, et al. Circulating miR-210 as a Novel Hypoxia Marker in Pancreatic Cancer. Transl Oncol 2010;3:109-13.

24. Resnick KE, Alder H, Hagan JP, Richardson DL, Croce CM, Cohn DE. The detection of differentially expressed microRNAs from the serum of ovarian cancer patients using a novel real-time PCR platform. Gynecol Oncol 2009;112:55-9.

25. Yamamoto Y, Kosaka N, Tanaka M, Koizumi F, Kanai Y, Mizutani T, et al. MicroRNA-500 as a potential diagnostic marker for hepatocellular carcinoma. Biomarkers 2009;14:529-38.

26. Wong TS, Liu XB, Wong BY, Ng RW, Yuen AP, Wei WI. Mature miR-184 as potential oncogenic microRNA of squamous cell carcinoma of tongue. Clin Cancer Res 2008;14:2588-92.

27. Feng G, Li G, Gentil-Perret A, Tostain J, Genin C. Elevated serum-circulating RNA in patients with conventional renal cell cancer. Anticancer Res 2008;28:321-6.

28. Ng EK, Chong WW, Jin H, Lam EK, Shin VY, Yu J, et al. Differential expression of microRNAs in plasma of patients with colorectal cancer: a potential marker for colorectal cancer screening. Gut 2009;58:1375-81.

29. Yu SL, Chen HY, Chang GC, Chen CY, Chen HW, Singh S, et al. MicroRNA signature predicts survival and relapse in lung cancer. Cancer Cell 2008;13:48-57.

30. Schetter AJ, Leung SY, Sohn JJ, Zanetti KA, Bowman ED, Yanaihara N, et al. MicroRNA expression profiles associated with prognosis and therapeutic outcome in colon adenocarcinoma. JAMA 2008;299:425-36.

31. Dong JT, Isaacs WB, Isaacs JT. Molecular advances in prostate cancer. Curr Opin Oncol 1997;9:101-7.

32. Meeker AK, Hicks JL, Platz EA, March GE, Bennett CJ, Delannoy MJ, et al. Telomere shortening is an early somatic DNA alteration in human prostate tumorigenesis. Cancer Res 2002; 62:6405-9.

33. Carver BS, Tran J, Gopalan A, Chen Z, Shaikh S, Carracedo A, et al. Aberrant ERG expression cooperates with loss of PTEN to promote cancer progression in the prostate. Nat Genet 2009;41:619-24.

34. Wang JC, Begin LR, Berube NG, Chevalier S, Aprikian AG, Gourdeau H, et al. Down-regulation of CD9 expression during prostate carcinoma progression is associated with CD9 mRNA modifications. Clin Cancer Res 2007;13:2354-61.

35. Massard C, Fizazi K. Targeting continued androgen receptor signaling in prostate cancer. Clin Cancer Res 2011;17:3876-83.

36. Coolen MW, Stirzaker C, Song JZ, Statham AL, Kassir Z, Moreno CS, et al. Consolidation of the cancer genome into domains of repressive chromatin by long-range epigenetic silencing (LRES) reduces transcriptional plasticity. Nat Cell Biol 2010;12:235-46.

37. Suh SO, Chen Y, Zaman MS, Hirata H, Yamamura S, Shahryari V, et al. MicroRNA-145 is regulated by DNA methylation and p53 gene mutation in prostate cancer. Carcinogenesis 2011;32:772-8

38. Sun R, Fu X, Li Y, Xie Y, Mao Y. Global gene expression analysis reveals reduced abundance of putative microRNA targets 
in human prostate tumours. BMC Genomics 2009;10:93.

39. Xu B, Feng NH, Li PC, Tao J, Wu D, Zhang ZD, et al. A functional polymorphism in pre-miR-146a gene is associated with prostate cancer risk and mature miR-146a expression in vivo. Prostate 2010;70:467-72.

40. Porkka KP, Pfeiffer MJ, Waltering KK, Vessella RL, Tammela TL, Visakorpi T. MicroRNA expression profiling in prostate cancer. Cancer Res 2007;67:6130-5.

41. Ozen M, Creighton CJ, Ozdemir M, Ittmann M. Widespread deregulation of microRNA expression in human prostate cancer. Oncogene 2008;27:1788-93.

42. Ambs S, Prueitt RL, Yi M, Hudson RS, Howe TM, Petrocca F, et al. Genomic profiling of microRNA and messenger RNA reveals deregulated microRNA expression in prostate cancer. Cancer Res 2008;68:6162-70.

43. Szczyrba J, Loprich E, Wach S, Jung V, Unteregger G, Barth S, et al. The microRNA profile of prostate carcinoma obtained by deep sequencing. Mol Cancer Res 2010;8:529-38.

44. Mattie MD, Benz CC, Bowers J, Sensinger K, Wong L, Scott GK, et al. Optimized high-throughput microRNA expression profiling provides novel biomarker assessment of clinical prostate and breast cancer biopsies. Mol Cancer 2006;5:24 .

45. Tong AW, Fulgham P, Jay C, Chen P, Khalil I, Liu S, et al. MicroRNA profile analysis of human prostate cancers. Cancer Gene Ther 2009;16:206-16.

46. Schaefer A, Jung M, Mollenkopf HJ, Wagner I, Stephan C, Jentzmik F, et al. Diagnostic and prognostic implications of microRNA profiling in prostate carcinoma. Int J Cancer 2010;126:1166-76.

47. Spahn M, Kneitz S, Scholz CJ, Stenger N, Rudiger T, Strobel P, et al. Expression of microRNA-221 is progressively reduced in aggressive prostate cancer and metastasis and predicts clinical recurrence. Int J Cancer 2010;127:394-403.

48. Martens-Uzunova ES, Jalava SE, Dits NF, van Leenders GJ Moller S, Trapman J, et al. Diagnostic and prognostic signatures from the small non-coding RNA transcriptome in prostate cancer. Oncogene 2012;31:978-91.

49. Cuzick J, Swanson GP, Fisher G, Brothman AR, Berney DM, Reid JE, et al. Prognostic value of an RNA expression signature derived from cell cycle proliferation genes in patients with prostate cancer: a retrospective study. Lancet Oncol 2011;12:245-55.

50. Penney KL, Sinnott JA, Fall K, Pawitan Y, Hoshida Y, Kraft P, et al. mRNA expression signature of Gleason grade predicts lethal prostate cancer. J Clin Oncol 2011;29:2391-6.

51. Lawrie CH, Gal S, Dunlop HM, Pushkaran B, Liggins AP, Pul- ford K, et al. Detection of elevated levels of tumour-associated microRNAs in serum of patients with diffuse large B-cell lymphoma. Br J Haematol 2008;141:672-5.

52. Yaman Agaoglu F, Kovancilar M, Dizdar Y, Darendeliler E, Holdenrieder S, Dalay N, et al. Investigation of miR-21, miR141 , and miR-221 in blood circulation of patients with prostate cancer. Tumour Biol 2011;32:583-8.

53. Brase JC, Johannes M, Schlomm T, Falth M, Haese A, Steuber $\mathrm{T}$, et al. Circulating miRNAs are correlated with tumor progression in prostate cancer. Int J Cancer 2011;128:608-16.

54. Waltering KK, Porkka KP, Jalava SE, Urbanucci A, Kohonen PJ, Latonen LM, et al. Androgen regulation of micro-RNAs in prostate cancer. Prostate 2011;71:604-14.

55. Mahn R, Heukamp LC, Rogenhofer S, von Ruecker A, Muller SC, Ellinger J. Circulating microRNAs (miRNA) in serum of patients with prostate cancer. Urology 2011;77:1265. e9-16.

56. Shen J, Hruby GW, McKiernan JM, Gurvich I, Lipsky MJ, Benson MC, et al. Dysregulation of circulating microRNAs and prediction of aggressive prostate cancer. Prostate 2012;72: 1469-77.

57. Bryant RJ, Pawlowski T, Catto JW, Marsden G, Vessella RL, Rhees B, et al. Changes in circulating microRNA levels associated with prostate cancer. Br J Cancer 2012;106:768-74.

58. Lodes MJ, Caraballo M, Suciu D, Munro S, Kumar A, Anderson B. Detection of cancer with serum miRNAs on an oligonucleotide microarray. PLoS One 2009;4:e6229.

59. Peltier HJ, Latham GJ. Normalization of microRNA expression levels in quantitative RT-PCR assays: identification of suitable reference RNA targets in normal and cancerous human solid tissues. RNA 2008;14:844-52.

60. Valadi H, Ekstrom K, Bossios A, Sjostrand M, Lee JJ, Lotvall JO. Exosome-mediated transfer of mRNAs and microRNAs is a novel mechanism of genetic exchange between cells. Nat Cell Biol 2007;9:654-9.

61. Arroyo JD, Chevillet JR, Kroh EM, Ruf IK, Pritchard CC, Gibson DF, et al. Argonaute2 complexes carry a population of circulating microRNAs independent of vesicles in human plasma. Proc Natl Acad Sci U S A 2011;108:5003-8.

62. Grange C, Tapparo M, Collino F, Vitillo L, Damasco C, Deregibus MC, et al. Microvesicles released from human renal cancer stem cells stimulate angiogenesis and formation of lung premetastatic niche. Cancer Res 2011;71:5346-56.

63. Mercatelli N, Coppola V, Bonci D, Miele F, Costantini A, Guadagnoli $\mathrm{M}$, et al. The inhibition of the highly expressed miR221 and miR-222 impairs the growth of prostate carcinoma xenografts in mice. PLoS One 2008;3:e4029. 